Research Article

$\mathrm{J}$ Exp Clin Med

2021; 38(2): 154-158

doi: $10.52142 /$ omujecm.38.2.18

\title{
Evaluation of toxoplasmosis risk on anxiety and perception of cat owner pregnant women
}

\author{
Nur Dokuzeylul GUNGOR ${ }^{1, *}$, Tuğba GÜRBÜZ ${ }^{2}$, Selma Bozkurt ZiNCİR ${ }^{3 \oplus}$, Banu DOKUZEYLÜL ${ }^{4} \oplus$, Erman OR ${ }^{4}$, \\ Ferhat CENGiz
}

\author{
${ }^{1}$ Department of Reproductive Endocrinology and IVF, BAU Medical Park Göztepe Hospital, Istanbul, Turkey \\ ${ }^{2}$ Department of Obstetrics and Gynecology, Medistate Hospital, Istanbul, Turkey \\ ${ }^{3}$ Private Office, Psychiatry, Istanbul, Turkey \\ ${ }^{4}$ Department of Internal Medicine, Faculty of Veterinary Medicine, Istanbul University-Cerrahpasa, Istanbul, Turkey \\ ${ }^{5}$ Embryology, BAU Medical Park Göztepe Hospital, Istanbul, Turkey
}

\begin{abstract}
\begin{tabular}{ccccc}
\hline Received: 07.09 .2020 & $\bullet$ & Accepted/Published Online: 16.12 .2020 & $\bullet$ & Final Version: 14.03 .2021 \\
\hline
\end{tabular}
Abstract

Cats are the main host of Toxoplasma gondii and pregnant women who own cats at home may be at risk. This condition may cause anxiety and stress in pregnant women. The aim of this study is to evaluate toxoplasmosis risk on anxiety and perception of cat owner pregnant women. The study group consisted of 59 pregnant women feeding cats in the home and 369 pregnant women not feeding cats. A Health Anxiety Inventory (HAI-18) was used to measure the anxiety of all participants. Age and anxiety variables were analyzed using a two-sample independent t-test. Gravida, education, and job status were analyzed using chi-square tests, and live to abort ratio \& pregnancy number were analyzed using Fisher exact test. Measurement of the anxiety level between the two groups showed that anxiety is significantly higher among the pregnant women who feed cats in their house with an average of $32.03 \pm 8.72$. The average anxiety level among the pregnant women who don't have cats in their house was $25.94 \pm 8.99$. The difference between the Health Anxiety Inventory of the two groups was significant $(p=0.0001)$ It was shown in the literature that pets can reduce anxiety but not studied in pregnant women, yet. Our results showed that pregnant women who own cats at home had more environmental anxiety than women who don't feed cats at home which is possibly associated with fear of Toxoplasma gondii transmission. This anxiety may also cause unfavorable pregnancy outcomes like eclampsia, preterm birth, low birth weight and high incidence of cesarean delivery.
\end{abstract}

Keywords: Anxiety, cat owner, pregnancy, toxoplasmosis

\section{Introduction}

Infectious diseases and various parasites threaten women's health during pregnancy, and this threat can also affect the health of the fetus by transferring it from mother to fetus (Dubey, 2004). Toxoplasmosis is one of the diseases caused by Toxoplasma gondii, a coccidial parasite whose final host is the cat, and its intermediate hosts are a wide range of warmblooded vertebrates (Dubey et al., 2006). In the United States it is estimated that $11 \%$ of the population 6 years and older have been infected with Toxoplasma (Robert-Gangneux and Dardé, 2012). In Turkey, Rize, Toxoplasma gondii IgM seropositivity was determined as $0.83 \%$ and $\operatorname{IgG} 33.64 \%$ in pregnant women (Gurlek and Colak, 2019). If a pregnant woman becomes infected shortly before or during pregnancy, she can transmit the disease to the fetus and it can cause serious complications in the fetus (Dubey, 2016). Infection of pregnant women with this disease occurs through cat feces directly or indirectly with any food contaminated with it (Dubey et al., 2006). Other examples of contamination include vegetables and fruits, eating raw or undercooked meat, kitchen utensils such as meat and cutlery used to prepare raw meat, and unpasteurized milk from livestock, especially goats (Jones, 2003).

There are three types of protozoan parasites in nature (Jones, 2001). 1) Tachyzoites, which has the form of an acute stage of the disease and the ability to multiply rapidly; 2) Bradyzoites, which is inside the tissue cysts and multiplies slowly; 3) Oocysts, which is only present in cat feces. In a period of one to three weeks, cats can excrete oocysts through feces, which keeps the parasite in its infectious form for more than a year (Perkins et al., 1973). The infection can be transmitted to the fetus throughout the pregnancy but the severity of complications decreases as the pregnancy advances 
(Ruskin et al., 1976).

The risk of fetal infection in the first 13 weeks of pregnancy is about $15 \%$, after which the risk of infection increases rapidly and reaches approximately $72 \%$ in week 36 (Weiss et al., 2009). Also, it was shown that the pregnant women seropositive to Toxoplasma IgG had similar mean MoM values of triple screening test parameters when compared to the pregnant women seronegative to Toxoplasma IgG antibody. (Gorkem et al., 2018). Complications of congenital toxoplasmosis are inversely related to fetal age so that the severity of the disease is higher in infants in the first trimester of pregnancy than at any other time. The risk of complications such as multiple organ failure severe neurological deficits such as hydrocephalus, microcephaly, mental retardation, and retinoid choroiditis, and even fetal death are higher in the first trimester of pregnancy (Wong et al., 1994). If an infection occurs in the third trimester of pregnancy, the baby is usually asymptomatic at birth and may show late symptoms such as chorioretinitis in the postpartum years (Frenkel, 1988).

Some people are very worried and anxious about their body and health without being at risk or suffering from a specific disease, and they consider simple physical symptoms as a serious illness. These people suffer from health anxiety (Avelino et al., 2003). Health anxiety is characterized by persistent and severe health concerns. In the diagnostic term, those who become disabled due to health anxiety are identified as hypochondriasis (Chaudhry et al., 2014). Health Anxiety is a new diagnosis in the latest US psychiatric classification that has partially replaced the patient's self-report (ElbezRubinstein et al., 2009).

On the other hand, pregnancy causes major physical and psychological changes in women. Fewer studies have been done on psychological changes during this period than on physical changes. Despite the development of biological therapies for medical problems during pregnancy and psychological problems such as anxiety and depression during this period, more research is still needed to clarify this issue from different aspects (Foulon et al., 1999).

Pregnancy is a potential stress-inducing process for mental health, increasing the severity of those who have previously had psychological problems (Garweg et al., 2005). Because cats are the main host of Toxoplasma gondii and can also play a role as intermediate hosts in the parasite's life cycle, pregnant women who own cats at home may be at risk. (Lappalainen, 1995; Gavinet, 1997; Kravetz, 2005). This condition can cause anxiety and stress in pregnant women. Therefore, this study evaluates the effect of feeding cats at home on anxiety and perception about toxoplasmosis in pregnant women.

\section{Materials and methods}

This study followed the principles of the Declaration of Helsinki and was approved by the Ethics Committee of Beykoz University. A total of 428 pregnant women followed in
Obstetrics and Gynecology clinics in Bahçeşehir University Faculty of Medicine and Private Medistate Hospital, were evaluated prospectively between July 2020 and September 2020.59 of the participants were feeding cats in the home (Group 1), and 369 of them were not feeding cats (Group 2). The participants cats were also referred to Department of Internal Medicine, Faculty of Veterinary Medicine, Istanbul University-Cerrahpaşa. Physical examinations were all done. All cats are healthy and don't show any symptoms of toxoplasmosis. For this reason, no toxoplasmosis tests are needed. Participants were divided into two groups according to whether they own cats at home or not. The groups were compared for the impact of the risk of getting toxoplasmosis on anxiety and perception. A Health Anxiety Inventory (HAI18) was used to measure the anxiety of participants. The questionnaire has three factors: Illness Likelihood, Illness Severity, and Body Vigilance (Salkovskis et al., 2002).

Three methods of analysis were used to analyze demographic data. Age and anxiety variables were analyzed using a two-sample independent t-test. Gravida, education, and job status were analyzed using chi-square tests, and Live to abort ratio \& pregnancy number were analyzed using Fisher exact test (more than $30 \%$ of cells have frequency lower than $5)$.

Descriptive statistics results will be given as mean \pm standard deviation or median (minimum-maximum) for numerical variables and number and/or percentage of patients for categorical variables. All continuous data are expressed as mean \pm standard deviation. The comparative analysis is made with the Mann-Whitney $U$ test for continuous data and $\chi 2$ test for categorical data. The ratio of categorical variables will be compared between groups using the chi-square test. $\mathrm{P}<0.05$ will be considered statistically significant. Patients with missing information or data on file were excluded from the study. SPSS statistical software for Windows (Statistical Package for Social Sciences, version 16.0, SPSS Inc. Chicago, Illinois, USA) was used to evaluate the study results

\section{Results}

The average age of the participants in this study was 29.95 $( \pm 3.93)$ years. Of our study population, $309(72.2 \%)$ experienced their first pregnancy, and another 119 (27.8\%) experienced their second or more pregnancy. More precisely, out of our study population, $309(72.2 \%)$ people experienced their first pregnancy, $56(13.08 \%)$ people experienced their second pregnancy, $49(11.45 \%)$ people experienced their third pregnancy, $12(2.8 \%)$ people experienced their fourth pregnancy, and $2(0.47 \%)$ people experienced their fifth pregnancy. In participants, 96 (82\%) had one, 19 (16.2\%) had two, and 2 had three $(1.8 \%)$ alive children. Also, among the participants, $54(96.6 \%)$ once and $2(1.8 \%)$ twice had experienced abortion. There were $109(25.47 \%)$ participants with high school education and $319(74.53 \%)$ participants with university education. Of the participants, 45 (10.51\%) were housewives, $252(58.88 \%)$ were employees in the solution sector, and $131(30.61 \%)$ were government officials. 
Results suggest that the two groups were not significantly different regarding demographic characteristics, and none of the 6 demographic variables showed significant differences between the two groups. So, we can assume that both groups were sampled from one statistical population.

Health Anxiety Env variable shows the result of Health Anxiety Inventory (HMI-18) questionnaire. Measurement of the anxiety level between the two groups showed that anxiety is significantly higher among the people who feed cats in their house with an average of $32.03( \pm 8.72)$. The average anxiety level among the people who don't feed cats in their house was 25.94 ( \pm 8.99$)$. As can be seen from Table 2, the difference between the Health Anxiety Inventory of the two groups was significantly different (P: 0.0001).

Table 1. Comparison of cat owners and not cat owners

\begin{tabular}{|c|c|c|c|}
\hline Group & $\begin{array}{l}\text { Cat Owners (Group:1) } \\
\qquad(\mathrm{n}=59)\end{array}$ & $\begin{array}{l}\text { Not Cat Owners (Group:2) } \\
\qquad(\mathrm{n}=369)\end{array}$ & P-value \\
\hline Age & $29.8( \pm 3.8)$ & $29.97( \pm 3.95)$ & 0.75 \\
\hline Primigravida, Multigravida & $46(78 \%), 13(22 \%)$ & $263(71.3 \%), 106(28.7 \%)$ & 0.29 \\
\hline Pregnancy number (1 to 5)* & $\begin{array}{l}46(78 \%), 7(11.9 \%), 5 \\
(8.5 \%), 1(1.7 \%), 0\end{array}$ & $\begin{array}{l}263(71.3 \%), 49(13.3 \%), 44 \\
(11.9 \%), 11(3 \%), 2(0.5 \%)\end{array}$ & 0.89 \\
\hline Live to abort ratio $(0,0.5,0.66,0.75,1)^{*}$ & $\begin{array}{l}0(0 \%), 5(38.5 \%), 1(7.7 \%) \\
0(0 \%), 7(53.8 \%)\end{array}$ & $\begin{array}{l}2(1.9 \%), 35(33 \%), 11(10.4 \%) \\
2(1.9 \%), 56(52.8 \%)\end{array}$ & 1.0 \\
\hline EDUCATION: High School, University & $11(18.6 \%), 48(81.4 \%)$ & $98(26.6 \%), 271(73.4 \%)$ & 0.195 \\
\hline $\begin{array}{l}\text { JOB STATUS (housewife, solution } \\
\text { sector, Government officials) }\end{array}$ & $\begin{array}{c}6(10.2 \%), 35(59.4 \%), 18 \\
(30.4 \%)\end{array}$ & $\begin{array}{c}39(10.6 \%), 217(58.8 \%), 113 \\
(30.6 \%)\end{array}$ & 0.99 \\
\hline Health Anxiety Env. & $32.03( \pm 8.72)$ & $25.94( \pm 8.99)$ & 0.0001 \\
\hline
\end{tabular}

\section{Discussion}

Antepartum depression, frequently in comorbidity with anxiety disorders, is a severe psychopathological condition frequently reported in pregnancy and in many cases associated with obstetric and neonatal complications and potential negative consequences on child neurodevelopment. The prevalence rates of depression during pregnancy have been reported as high as $7.4 \%$ in the first trimester and $12.0-12.8 \%$ in the second and third trimester with even higher rates in the first year after delivery (Howdeshell and Orney, 2017). Untreated maternal depression can have adverse consequences for both the patient and her offspring. Stress and anxiety during pregnancy are associated with consequences such as preterm labor, low birth weight, respiratory problems, and depression (Berrebi et al., 1994). In a study of 357 pregnant women, Schetter and Tanner (Schetter et al., 2012) showed that 54\% of the participants had symptoms of anxiety, and $37 \%$ of them had symptoms of depression. A study by Kurki, (Kurki et al., 2000) on 623 pregnant women showed that the presence of signs of depression and anxiety in pregnant women is a risk factor for eclampsia. In a study of 330 pregnant women, Neshat, Majlesi (Neshat et al., 2013) reported that the risk of preterm birth in those with anxiety, stress, and depression was 3.7 times that of normal individuals. Bahrami et al. (Bahrami et al., 2013) in a study of 180 pregnant women reported that anxiety and depression during pregnancy increased the risk of cesarean delivery by 2.3 times, as well as the risk of low birth weight in pregnant women with stress, was 3.1 times higher than stressfree pregnant women. The degree of severity of maternal depression, social and reproductive risk factors, obesity, and stressful events may further exacerbate this effect. Newborns of depressed mothers actually showed poor habituation, orientation, motor, range of state, autonomic stability and higher depressive scores at Brazelton Scale (Field et al., 2004). Moreover, newborns of depressed mothers had higher cortisol levels and lower serotonin (5-HT) levels.

Our results showed that pregnant women who feed cats at home had, on average, more environmental anxiety than women who do not feed cats at home. As far as we know, previous studies have not examined the anxiety of pregnant women about their cats concerning toxoplasmosis, so the results of our study cannot be directly compared with other studies. However, most studies have shown that keeping pets can reduce anxiety (Muschel,1984; Friedmann et al., 1995; Kaminski et al., 2002; Buttelmann, 2014; Gadomski et al., 2015; Wright et al., 2015). Previous studies have shown that people who have pets can more easily cope with severe emotional problems such as bereavement (Friedmann, 2013) and have higher emotional health (Friedmann, 1995). However, studies haven't shown a positive relationship between animal care and mental health (Beetz et al., 2012).

Mugford, M'Comisky (Mugford, 1975), in their study of the elderly, showed that giving birds to these people increased their mental health over six months. Serpell (Serpell, 1991) also found in a study that people with pets showed fewer psychological problems than those in the control group. Feeding animals can also boost people's well-being, create a non-threatening relationship, and meet many of their emotional needs so that people who have pets or deal with animals have 
less stress and anxiety. They report fewer depressive symptoms and better physical health (Mugford, 1975). However, Straede and Gates (Straede et al., 1993) concluded that there is no difference between pet owners and non-pet owners in terms of psychological health.

The mental health benefits of pet companionship compared to non-pet ownership is well documented, but there are contrary results as well. The results of a study showed that cat owners rated their symptoms of depression to a higher degree than both dog and non-pet owners (Connell et al., 2007).

In this study, we found a significant difference between the environmental anxiety of women who feed cats and women who do not feed cats, which is inconsistent with the results of similar studies. The reason for this inconsistency is because cats are the main hosts for Toxoplasma gondii and can also act as intermediate hosts in the parasite's life cycle, and women who pet cats at home may be at risk and thinking about this risk will increase their anxiety. In other studies, pets are often seen as emotional and psychological supporters (Muschel, 1984; Friedmann et al., 1995; Kaminski et al., 2002; Buttelmann, 2014; Gadomski et al., 2015; Wright et al., 2015). Still, in our case, pets, and cats in particular, are considered to be the risk factors for Toxoplasma gondii. Instead of providing emotional support and comfort to the owner, this risk factor raises concerns about parasite transmission to the mother and potential harm to the fetus. The low number of patients and retrospective study design were among the limitations of our study. To the best of our knowledge, this is the first study in the literature investigating the toxoplasmosis risk on anxiety and perception of cat owner pregnant women.

Therefore, it can be concluded that in this particular case, cats as pets, due to the potential risk they pose to pregnant women, have a negative role in causing anxiety and perception in pregnant women.

In this study, we looked at the effect of feeding a cat at home on anxiety and worry about toxoplasmosis in pregnant women. Our results showed that pregnant women who feed cats at home had, on average, more environmental anxiety than women who did not feed cats at home. The reason for this anxiety can be considered the fear of transmitting Toxoplasma gondii from cat to mother and its potential harm to the fetus. Contrary to the usual supportive role of pets, this particular study showed that cats can increase the anxiety of pregnant women. This contradiction reminds us that in addition to examining the physiological effects of parasites or diseases, it is important to examine the psychological effects of their fear and anxiety and the cats' physical condition. Teaching women to avoid infection during pregnancy offers the most practical preventive approach. There is little doubt that, if pregnant women followed a few simple guidelines in their cat, food and personal hygiene behaviors, the risk of congenital toxoplasmosis could be greatly reduced or eliminated.

\section{Conflict of Interest}

The author declares that there is no conflict of interest.

\section{Acknowledgements}

Thank all of the project participants for their contributions.

\section{References}

1. Avelino, M.M., Campos Jr, D., de Parada, J.C.D., de Castro, A.M., 2003. Pregnancy as a risk factor for acute toxoplasmosis seroconversion. Eur. J. Obstet. Gynecol. Reprod. Biol. 108 (1), 1924.

2. Bahrami, N., Bahrami, S., 2013. Correlation between prenatal depression with delivery type and neonatal anthropometric indicators. Koomesh, 15(1), 39-45.

3. Beetz, A., Uvnäs-Moberg, K., Julius, H., Kotrschal, K. 2012. Psychosocial and psychophysiological effects of human-animal interactions: the possible role of oxytocin. Front. Psychol. 3, 234.

4. Berrebi, A., Kobuch, W.E., Bessieres, M.H., Bloom, M.C., Rolland, M., Sarramon, M.F., Roques, C., Fournié, A., 1994 Termination of pregnancy for maternal toxoplasmosis. Lancet. 344 (8914), 36-39.

5. Buttelmann, D., Römpke, A.K. 2014. Anxiety-reducing effect: Dog, fish and plant in direct comparison. Anthrozoös, 27(2), 267277.

6. Chaudhry S.A., Gad, N., Koren, G., 2014. Toxoplasmosis and pregnancy. Can. Fam. Physician., 60 (4), 334-336.

7. Connell, C.M., Janevic, M.R., Solway, E., McLaughlin, S.J., 2007. Are pets a source of support or added burden for married couples facing dementia? J. Appl. Gerontol. 26(5), 472-485.

8. Dubey, J., 2004. Toxoplasmosis-a waterborne zoonosis. Vet Parasitol. 126(1-2), 57-72.

9. Dubey, J., Lappin M. 2006. Toxoplasmosis and neosporosis. Infectious diseases of the dog and cat, 2, 493-509.

10. Dubey, J.P., Toxoplasmosis of animals and humans. 2016: CRC press.

11. Elbez-Rubinstein, A., Ajzenberg, D., Dardé, M.L., Cohen, R., Dumètre, A., Year, H., Gondon, E., Janaud, J.C., Thulliez, P. 2009. Congenital toxoplasmosis and reinfection during pregnancy: case report, strain characterization, experimental model of reinfection, and review. J. Infect. Dis. 199 (2), 280-285.

12. Field, T., Diego, M., Dieter, J., 2004. Prenatal depression effects on 656 the fetus and the newborn. Inf. Behav. Develop. 27, 216229.

13. Foulon, W., Villena, I., Stray-Pedersen, B., Decoster, A., Lappalainen, M., Pinon, J.M., Jenum, P.A., Hedman, K., Naessens, A. 1999. Treatment of toxoplasmosis during pregnancy: a multicenter study of impact on fetal transmission and children's sequelae at age one year. Am. J. Obstet. Gynecol. 180 (2), 410415.

14. Frenkel, J., 1988. Pathophysiology of toxoplasmosis. Parasitol. Today. 4(10), 273-278.

15. Friedmann, E., The role of pets in enhancing human well-being: physiological. The Waltham book of human-animal interaction: Benefits and responsibilities of pet ownership, 2013. 33.

16. Friedmann, E. and S.A. Thomas, Pet ownership, social support, and one-year survival after acute myocardial infarction in the Cardiac Arrhythmia Suppression Trial (CAST). The American journal of cardiology, 1995. 76 (17), 1213-1217.

17. Gadomski, A.M., Scribani, M.B., Krupa, N., Jenkins, P., 
Nagykaldi, Z., Olson, A.L., 2015. Peer Reviewed: Pet Dogs and Children's Health: Opportunities for Chronic Disease Prevention? Preventing chronic disease, 12.

18. Garweg, J.G., Scherrer, J., Wallon, M., Kodjikian, L., Peyron, F., 2005. Reactivation of ocular toxoplasmosis during pregnancy. BJOG. 112(2): 241-242.

19. Gavinet, M.F., Robert, F., Firtion, G., Delouvrier, E., Hennequin, C., Maurin, J.R., Tourte-Schaefer, C., Dupouy-Camet, J. 1997. Congenital toxoplasmosis due to maternal reinfection during pregnancy. J. Clin. Microbiol., 35(5):1276-1277.

20. Gorkem, U., Gureser, A., Togrul, C., Karasartova, D., Gungor, T., Ozkan, A., Kocak, O., 2018. Influence of Maternal Toxoplasmosis on the Second-Trimester Aneuploidy Screening Test. Obstet. Gynaecol. Reprod. Med. 24 (2), 71-75.

21. Gurlek, B., Colak, S., 2019. Antenatal Toxoplasma gondii, Rubella, and Cytomegalovirus Infection Screening Among Pregnant Women Attending Tertiary University Hospital. Obstet. Gynaecol. Reprod. Med. 25(2), 74-80.

22. Howdeshell, K.L., Ornoy, A., 2017. Depression and Its Treatment During Pregnancy: Overview and Highlights._Birth Defects Res. 17;109(12):877-878.

23. Jones, J., Lopez, A., Wilson, M., 2003. Congenital toxoplasmosis. Am. Fam. Physician, 67(10): 2131-2138.

24. Jones, J.L., Lopez, A., Wilson, M., Schulkin, J., Gibbs, R., 2001. Congenital toxoplasmosis: a review. Obstet. Gynecol. Surv. 56(5): 296-305.

25. Kaminski, M., Pellino, T., Wish, J., 2002. Play and pets: The physical and emotional impact of child-life and pet therapy on hospitalized children. Child. Health Care. 31(4): 321-335.

26. Kravetz, J.D. and D.G. Federman, Prevention of toxoplasmosis in pregnancy: knowledge of risk factors. Infectious diseases in obstetrics and gynecology, 2005. 13(3): p. 161-165.

27. Kurki T, Hiilesmaa V, Raitasalo R, Mattila H, Ylikorkala O. Depression and anxiety in early pregnancy and risk for preeclampsia. Obstetrics \& Gynecology, 2000. 95(4): p. 487-490.

28. Lappalainen, M., Sintonen, H., Koskiniemi, M., Hedman, K., Hiilesmaa, V., Ammälä, P., Teramo, K., Koskela, P., 1995. Costbenefit analysis of screening for toxoplasmosis during pregnancy. Scandinavian journal of infectious diseases, 27(3): 265-272.

29. Mugford, R., J. M'Comisky, R. Anderson, Pet animals and society. 1975, Bailliere Tindall London.

30. Muschel, I.J., 1984. Pet therapy with terminal cancer patients.
Social Casework. 65(8): p. 451-458.

31. Neshat, R. Investigation the relationship between preterm delivery and prevalence of anxiety, stress and depression in pregnant women of dorrod health center, Iran in 2010. IJOGI. 2013. 16(67): p. 16-24.

32. Perkins, E., Ocular toxoplasmosis. The British journal of ophthalmology,1973. 57(1): p. 1.

33. Robert-Gangneux F., Dardé, M.L., 2012. Epidemiology of and diagnostic strategies for toxoplasmosis. Clin. Microbiol. Rev. 25, 264-296.

34. Ruskin, J., Remington, J.S., 1976. Toxoplasmosis in the compromised host. Annals of Internal Medicine, 84(2): 193-199.

35. Salkovskis PM, Rimes KA, Warwick HM, Clark DM., 2002. The Health Anxiety Inventory: development and validation of scales for the measurement of health anxiety and hypochondriasis. Psychol Med. 32(5), 843-853.

36. Dunkel Schetter, C., Tanner, L.,2012. Anxiety, depression and stress in pregnancy: implications for mothers, children, research, and practice. Curr. Opin. Psychiatry. 25(2), 141

37. Serpell, J., 1991. Beneficial effects of pet ownership on some aspects of human health and behaviour. J. R. Soc. Med., 84(12): 717-720.

38. Straede, C.M., Gates, R.G., 1993. Psychological health in a population of Australian cat owners. Anthrozoös, 6(1): 30-42.

39. Weiss, L.M, Dubey, J.P., 2009. Toxoplasmosis: a history of clinical observations. International journal for parasitology, 39(8), 895-901.

40. Wong, S.Y., Remington, J.S. 1994. Toxoplasmosis in pregnancy. Clinical infectious diseases, 853-861.

41. Wright, H., 2015. Pet dogs improve family functioning and reduce anxiety in children with autism spectrum disorder. Anthrozoös, $28,611-624$. 